\title{
Age-dependent redistribution and hypersialylation of the central myelin paranodal loop membrane protein Opalin in the mouse brain
}

Yumi Sato $^{1,+, \#}$, Fumio Yoshikawa ${ }^{1, \#}$, Tetsushi Sadakata ${ }^{1,2}$, Yo Shinoda ${ }^{1,3}$, Michinori Koebis $^{3}$, and Teiichi Furuichi ${ }^{1,3, *}$

1 Laboratory for Molecular Neurogenesis, RIKEN Brain Science Institute, Saitama 351-0198, Japan, ${ }^{2}$ Advanced Scientific Research Leaders Development Unit, Gunma University, Gunma 371-8511, Japan, ${ }^{3}$ Department of Applied Biological Science, Faculty of Science and Technology, Tokyo University of Science, Chiba 278-8510, Japan

* Address correspondence to: Teiichi Furuichi, Department of Applied Biological Science, Faculty of Science and Technology, Tokyo University of Science, 2641 Yamazaki, Noda, Chiba 278-8510, Japan. Tel: +81-4-7122-9303; Fax: +81-4-7123-9767; E-mail: tfuruichi@rs.tus.ac.jp

+ Current address: Graduate School of Medical Science, Department of Molecular Virology and Oncology, Cancer Research Institute, Kanazawa University, Kanazawa, Ishikawa, Japan

\# These authors are contributed equally to this work. 


\section{Abstract}

Opalin/Tmem10 is a myelin-associated sialylglycoprotein that is specific to only the mammalian central nervous system. However, little is known about the properties or function of this protein. Here, we analyzed the expression and glycosylation patterns of Opalin in the postnatal mouse brain. Immunolocalization patterns of Opalin were similar to those of myelin basic protein in juvenile and adolescent mice. On the other hand, in the adult mouse brain, decreasing immunoreactivity for Opalin was observed in the hindbrain region especially in the cerebellar white matter compared with the corpus callosum in the forebrain. In addition, Opalin showed increasing molecular size with mouse aging. This age-dependent increase in Opalin molecular weight was mainly owing to hypersialylation of $O$-glycans. These results indicate that the regional redistribution and the degree of sialylation of Opalin protein are age-dependently regulated in mouse brains. 


\section{Introduction}

Opalin/Tmem10 is a myelin protein phylogenetically specific to mammals and specifically localized to the central nervous system but not the peripheral nervous system [1], [2], [3], [4] and [5]. We previously showed that Opalin is localized at high levels in the "piled-up" myelin paranodal loops as well as the soma and processes of oligodendrocytes [5]. Because of these features, Opalin is often used as a good marker for mature oligodendrocytes and myelin of the mammalian CNS [6], [7] and [8]. It is another intriguing feature that Opalin, a type I transmembrane protein (143 amino acids), has a short $\mathrm{N}$-terminal 30 amino-acid domain that undergoes a high degree of post-translational modification by $N$-linked and $O$-linked oligosaccharides, some of which are terminated with sialic acids [5]. Mutations at several consensus or putative sites for $\mathrm{N}$-glycan and $\mathrm{O}$-glycan sites caused Opalin a failure of cell surface localization in oligodendrocytes, suggesting that the large sialylated glycans of the N-terminal domain is critical for Oplain protein trafficking and/or function in paranodal loop membrane [5]. However, glycosylation patterns of Opalin protein in the brain are not fully understood.

In the present study, we investigated expression and glycosylation of Opalin protein in mouse brain during postnatal development and aging. We found age-dependent changes in brain distribution patterns and degree of sialylation of Opalin protein. The expressional and post-translational alteration of Opalin from juvenile stages to adulthood suggests its possible role in the myelin of mammalian brains. 


\section{Materials and Methods}

\subsection{Animals}

Mice (ICR and C57BL/6J) were purchased from Nihon SLC (Hamamatsu, Japan). All animal work was conducted according to the recommendations and the protocols approved by the Animal Care and Use Committee of RIKEN and Tokyo University of Science (approval number: H21-2-244(4) and N13016, respectively). Animals were housed in an environment with a 12:12-hour light/dark cycle (daytime 8:00-20:00) with controlled temperature $\left(23 \pm 2^{\circ} \mathrm{C}\right)$ and humidity $(55 \pm 10 \%)$ and ad libitum access to food and water.

\subsection{Antibodies}

The rabbit anti-mouse Opalin antibody was generated in a previous study [5] (catalog no. RIK-B-OP, Cosmo Bio Co., LTD) and used at a concentration of $1 \mu \mathrm{g} / \mathrm{ml}$. The anti-myelin basic protein (MBP) (1:10000 for WB and 1:2000 for IHC, rabbit polyclonal, Cat. 16141) was obtained from IBL, Gunma, Japan. The secondary antibodies used were horseradish peroxidase (HRP)-conjugated anti-rabbit IgG (H+L) (1:2000) (Cat. NA9340) and HRP-conjugated anti-mouse $\operatorname{IgG}(\mathrm{H}+\mathrm{L})$ (1:2000) (Cat. NA9310) from GE Healthcare, Chalfont St. Giles, UK. The secondary antibody used for diaminobenzidine staining was biotinylated anti-rabbit IgG (1:1000) (Cat. BA-1000, from Vector, Burlingame, CA).

\subsection{Subcellular fractionation}


Subcellular fractionation was carried out as reported in previous studies [5] and [9]; details are provided in the Supplemental materials. Briefly, mouse brain homogenates were prepared using a grass-Teflon homogenizer and were centrifuged at $1,000 \times g$ for $10 \mathrm{~min}$ at $4^{\circ} \mathrm{C}$ to obtain precipitate fraction 1 (ppt1). The resulting supernatant (called sup 1 fraction) was centrifuged at $105,000 \times g$ for $1 \mathrm{~h}$ at $4^{\circ} \mathrm{C}$ to obtain the precipitate fraction $2+3(\mathrm{ppt} 2+3)$ containing crude cell membrane [5] and [9].

Myelin fractions were prepared essentially as described [5] and [10]; the detailed method is provided in the Supplemental materials. Briefly, mouse brain homogenates were prepared with a Dounce homogenizer. The homogenates were layered over $0.85 \mathrm{M}$ sucrose solution in 5mM Tris- $\mathrm{HCl}(\mathrm{pH} 7.4)$ in a Beckman Coulter SW28 rotor and were centrifuged at $75,000 \times g$ for $30 \mathrm{~min}$ at $4^{\circ} \mathrm{C}$. The crude myelin layer at the interface of the two sucrose solutions was collected and recentrifuged in a SW28 rotor at 75,000 $\times g$. The pellet was resuspended and was recentrifuged at $12,000 \times g$. The resultant pellet was again homogenized and repelleted at $12,000 \times g$ to obtain the crude myelin fraction. The crude myelin membrane pellet obtained was homogenized, layered over $0.85 \mathrm{M}$ sucrose solution in $5 \mathrm{mM}$ Tris- $\mathrm{HCl}(\mathrm{pH} 7.4)$, and centrifuged in a SW28 rotor at 75,000 $\times g$ for $15 \mathrm{~min}$ at $4^{\circ} \mathrm{C}$. The myelin fraction at the interface between $0.35 \mathrm{M}$ and $0.85 \mathrm{M}$ sucrose solution was collected, again homogenized, and recentrifuged in a SW28 rotor as described above. The resulting pellet was used as the pure myelin fraction. 


\subsection{Immunological analysis}

Western blot analyses of protein samples and immunohistochemical analyses of brain sections (number of animals analyzed=3) were basically carried out as described previously [5] and [9]; the details are provided in the Supplemental materials.

\subsection{In situ hybridization}

In situ hybridization of mouse brain and spinal cord sections (number of animals analyzed=3) was carried out as described in previous studies [5] and [9].

\subsection{Enzymatic deglycosylation}

The deglycosylation reaction was essentially performed as described previously [5], and the detail is provided in Supplemental materials. $N$-linked glycans were removed by treatment with $\mathrm{N}$-glycosidase F (from Flavobacterium meningosepticum) (Roche Diagnostics GmbH, Mannheim, Germany), $O$-linked glycans were removed by incubation with $O$-glycosidase (from Diplococcus pneumoniae) (Roche), and Sialic acids were removed by incubation with neuraminidase (from Arthrobacter ureafaciens) (Roche).

\subsection{Lectin affinity assay}

The lectin-binding affinity of Opalin in myelin fractions at P21 and P180 was analyzed using Maackia amurensis lectin II (MAL II) (Cat. B-1265, from Vector Laboratories, Inc., 
Burlingame, CA), Maackia amurensis lectin (MAA) (Cat. BA-7801-2, from EY Laboratories, Inc., San Mateo, CA) and Sambucus nigra lectin (SNA) (Cat. BA-6802-1, from EY Laboratories, Inc.). The detailed method is provided in the Supplemental materials.

\section{Results}

\subsection{Increase in the molecular size of Opalin protein with aging}

We examined Opalin protein in the crude cell membrane fraction ppt $2+3$ prepared from mouse cerebellum and cerebrum at five different postnatal stages (postnatal days [P] 7, P14, P28, P56 and P180) by western blot analyses using anti-Opalin antibody (Fig. 1A). Opalin immunoreactive protein was barely detectable in the cerebellar and cerebral lysates prepared at P7 (infant stage). The amount of Opalin protein increased dramatically in the cerebellum by P14 and became obvious in the cerebrum between P14 and P28 (juvenile stage). Notably, the bands immunopositive for Opalin in both cerebellum and cerebrum at P56 (adolescent stage) and P180 (adult stage) migrated more slowly in $12 \%$ SDS-PAGE than those at early stages (Fig. 1A). $15 \%$ SDS-PAGE of pure myelin fraction samples more convincingly separated Opalin bands at P23 from bands at P180 (Fig. 1B and C). These results suggest an increase in molecular size of Opalin with mouse aging, for example due to post-translational modification or splicing variation. 


\subsection{Change in region-specific expression patterns of Opalin with aging}

We next observed the localization of Opalin in sagittal (Fig. 2A-H) and coronal (Fig. 2I-N) sections of the mouse brain at four different postnatal stages by immunohistochemistry. Expression of Opalin protein was barely detectable over the mouse brain regions at P7 (Fig. 2A), rapidly increased by P14 (Fig. 2B), and almost reached peak levels around P21 across the white matter rich regions including corpus callosum, hippocampal fimbria, medullary stria of thalamus, anterior commissure, optic nerve, cerebellar white matter, corticospinal tract, and posterior funiculus (Fig. 2C), as previously described [5]. By contrast, expression of myelin basic protein (MBP), a major myelin protein, was already detectable at P7 (Fig. 2E) and was progressively increased thereafter in similar Opalin-positive regions (Fig. 2F and G). At P180 Opalin immunoreactivity, however, was substantially reduced in many regions of the mid-hind brain, especially the white matter of the cerebellum (Fig. 2D and I-K) compared with MBP-immunoreactivity (Fig. 2H and L-N). However the anterior commissure, corpus callosum, corticospinal tract, fasciculus retroflexus, fimbria of hippocampus, lateral olfactory tract, medullary stria of thalamus, optic chiasm, and optic tract retained relatively high levels (Fig. 2D) comparable with those in P21 old animals (Fig. 2C). This tendency of region-specific Opalin reduction in P180 mouse brains was supported by the postnatal changes of Opalin protein contents detected by western blot analysis (Fig. 1) and Opalin mRNAs analyzed by the GeneChip array (Supplemental Fig. $1)$. 
In the spinal cord, Opalin immunoreactivity was slightly higher in the white matter than the gray matter at P15 (Fig. 3A). At P180, the immunoreactivity was predominantly localized in the corticospinal tracts and became higher in the gray matter than the white matter (Fig. 2D and Fig. 3A). Such a region-specific change in P180 mouse spinal cords was not observed in MBP immunoreactivity (Fig. 2H and Fig. 3A). Regarding expression of Opalin in the gray matter, positive-signal patterns for Opalin proteins (Fig. 3B) and mRNAs (Fig. 3C) in the gray matter were almost comparable with those of MBP, suggesting that Opalin is expressed in cells (probably oligodendrocytes) of the gray matter in adults.

\subsection{Hyper-sialylation of Opalin with aging}

Endogenous Opalin (calculated molecular mass of $16 \mathrm{kDa}$ ) is immunodetected as a slowly-migrating broad band at approximately $33-38 \mathrm{kDa}$ in SDS-PAGE because it is highly glycosylated [5]. We compared glycosylation of myelin Opalin at P23 and P180 by enzymatic deglycosylation with $O$-glycosidase, neuraminidase and $N$-glycosidase F (Fig. 4A). Banding patterns of immunopositive Opalin were much broader at P23 (lane 1) than P180 (lane 2). The Opalin bands were apparently not changed after treatment with $O$-glycosidase (lanes 3 and 4), which cannot cleave sialylated forms of $O$-glycans, but they were shifted to almost the same molecular size after treatment with $O$-glycosidase and neuraminidase (lanes 5 and 6), suggesting that Opalin $O$-glycans at P23 and P180 were sialylated. Only neuraminidase treatment at P23 and P180 (lanes 7 and 8) gave rise to the 
bands that migrated a little more slowly than those treated with $O$-glycosidase and neuraminidase (lanes 5 and 6), suggesting that Opalin at P23 and P180 had very small $O$-glycan residues. Removal of both sialic acids and $O$-glycans (lanes 5 and 6) or both sialic acid and $N$-glycans (lanes 9 and 10) did not generate large differences in mobility of Opalin bands between $\mathrm{P} 23$ and P180, suggesting that $N$-linked and $O$-linked glycosylation in Opalin was not greatly changed with aging. On the other hand, removal of $N$-glycans (lanes 11 and 12) or both $N$-glycans and non-sialylated $O$-glycans (lanes 13 and 14) exhibited multiple bands in P23 old animals but at least two bands (a major and a minor bands) at P180, suggesting that there is a variation in the length of sialic acid residues attached to $O$-glycans at P23 but almost constant length of these residues at P180. Removal of sialic acids and $\mathrm{N}$-linked and $\mathrm{O}$-linked glycans gave rise to at least two bands at either P23 or P180 (lanes 15 and 16).

We finally estimated the properties of sialylated $O$-glycans of Opalin in juvenile and adult mouse brains by a lectin-binding assay (Fig. 4B). The results showed that glycans of Opalin at both P23 and P180 were mostly terminated by sialic acid- $\alpha 2,3$-galactose- $\beta 1$, 3-N-acetylgalactosamine ( $\mathrm{Sia}[\alpha 2,3] \mathrm{Gal}[\beta 1,3] \mathrm{GalNAc})$ (affinity for MAL II) and also by sialic acid- $\alpha 2,6$-galactose/N-acetylgalactosamine ( $\mathrm{Sia}[\alpha 2,6] \mathrm{Gal} / \mathrm{GalNAc})$ (affinity for SNA). Opalin did not bind to MAA which recognizes $\operatorname{Sia}(\alpha 2,3) \mathrm{Gal}(\beta 1,4) \mathrm{GlcNAc}$ (affinity for MAA, see Supplemental materials). Immunopositive band patterns of Opalin affinity-purified for both MAL II and SNA were broad at P23 but very sharp at P180, and 
more intense bands for MAL II affinity was observed at P180 than P23. Molecular size of deglycosylated Opalin bands did not differ between samples at P23 and P180 (Fig. 4A, lanes 15 and 16).

Altogether, the deglycosidase treatment experiments suggested that increasing complexity of carbohydrates, mainly increasing sialylation of $O$-glycans with at least $\operatorname{Sia}(\alpha 2,3) \operatorname{Gal}(\beta 1,3) \mathrm{GalNAc}$ and $\operatorname{Sia}(\alpha 2,6) \mathrm{Gal} / \mathrm{GalNAc}$ linkages, is a major factor in the increasing molecular size of Opalin with aging, and reaches almost a plateau by P180. In addition, alteration(s) of the $\mathrm{N}$-linked and $\mathrm{O}$-linked glycosylation and alternative splicing, which may cause protein size variations, are not directly associated with the size difference of Opalin between P23 and P180.

\section{Discussion}

In the present study, we examined the regional expression and post-translational modification of myelin sialylglycoprotein Opalin in the mouse brain. We found a region-specific change of Opalin expression in mouse brains from infant to adult stages. The expression level of Opalin was dramatically decreased with age in the white matter of the cerebellum, but most of the other brain regions such as the corpus callosum, corticospinal tract, hippocampal fimbria, medullary stria of thalamus, anterior commissure, and optic nerve did not show such drastic changes in expression levels. Our results also 
indicated that the molecular size of Opalin is gradually increased in adult mouse brains. The molecular basis of this size increase was identified as post-translational modification owing to an increasing sialylation of $O$-glycans with age.

Although a detailed structural analysis of Opalin glycans is needed to understand the biological significance of age-dependent hypersialylation, our results suggest that Opalin contains $O$-glycans terminated by sialic acid moieties with at least $\operatorname{Sia}(\alpha 2,3) \mathrm{Gal}(\beta 1,3) \mathrm{GalNAc}$ and $\operatorname{Sia}(\alpha 2,6) \mathrm{Gal} / \mathrm{GalNAc}$ linkages and that the former sialic acid linkage become more dominant in P180 mouse brains compared with P23 brains. Negatively-charged sialic acids are known to provide hydrophilicity to cell surfaces, leading to regulation of cellular interactions [11], [12], [13] and [14]. Our previous study showed that Opalin has at least three glycan sites (two $N$-glycan sites [Asn-6 and Asn-12] and an $O$-glycan site [Thr-14]) [5] and that mutations of these glycan sites fail to localize at cell surfaces in primary cultured oligodendrocytes [5]. Therefore, we hypothesize that Opalin may play a role in intermembranous interaction between paranodal loops, intercellular signaling between oligodendrocytes, or oligodendrocyte-astrocyte signaling via the sialic acid moiety. It is also intriguing that many pathogens recognize sialic acids on the cell surface: for example, the human polyomavirus JC virus can infect oligodendrocytes by interacting with the terminal $\alpha 2,6$-linked sialic acids of the cell surface receptor, resulting in the fatal demyelinating CNS disease [15]. Further studies in pathogenic as well as non-pathogenic state may provide a clue for understanding Opalin function in the 
mammalian CNS myelin.

In conclusion, our study demonstrates that the regional expression pattern and the sialylation pattern of the transmembrane sialylglycoprotein Opalin is regulated during development. These age-dependent alterations of Opalin protein may be associated with its role in the maturation, maintenance and/or aging of the mammalian central myelin.

\section{Acknowledgements}

This study was supported by Grants from the Japanese Ministry of Education, Culture, Sports, Science and Technology, the Japan Society for the Promotion of Science, the Japan Science and Technology Agency, the Hamaguchi Foundation for the Advancement of Biochemistry, and the Tokyo University of Science. 


\section{References}

1. Aruga J, Yoshikawa F, Nozaki Y, Sakaki Y, Toyoda A, Furuichi T (2007) An oligodendrocyte enhancer in a phylogenetically conserved intron region of the mammalian myelin gene Opalin. J. Neurochem. 102: 1533-1547.

2. Golan N, Adamsky K, Kartvelishvily E, Brockschnieder D, Mobius W, Spiegel I, Roth AD, Thomson CE, Rechavi G, Peles E (2008) Identification of Tmem10/Opalin as an oligodendrocyte enriched gene using expression profiling combined with genetic cell ablation. Glia 56: 1176-1186.

3. Kippert A, Trajkovic K, Fitzner D, Opitz L, Simons M (2008) Identification of Tmem10/Opalin as a novel marker for oligodendrocytes using gene expression profiling. BMC Neurosci. 9: 40.

4. Dugas JC, Tai YC, Speed TP, Ngai J, Barres BA (2006) Functional genomics analysis of oligodendrocyte differentiation. J. Neurosci. 26: 10967-10983.

5. Yoshikawa F, Sato Y, Tohyama K, Akagi T, Hashikawa T, Nagakura-Takagi Y, Sekine Y, Morita N, Baba H, Suzuki Y, Sugano S, Sato A, Furuichi T (2008) Opalin, a transmembrane sialylglycoprotein located in the central nervous system myelin paranodal loop membrane. J. Biol. Chem. 283: 20830-20840.

6. Haines JD, Fang J, Mushynski WE, Almazan G (2010) Mitogen-activated protein kinase activated protein kinase 2 (MK2) participates in p38 MAPK regulated control of oligodendrocyte differentiation. Glia 58: 1384-1393. 
7. Tripathi RB, Rivers LE, Young KM, Jamen F, Richardson WD (2010) NG2 glia generate new oligodendrocytes but few astrocytes in a murine experimental autoimmune encephalomyelitis model of demyelinating disease. J. Neurosci. 30: 16383-16390.

8. Fletcher JL, Kondarari GS, Wright AL, Thomson PC, Williamson P, Taylor RM (2011) Myelin genes are downregulated in canine fucosidosis. Biochimica Biophysica Acta 1812: $1418-1426$.

9. Yoshikawa F, Iwasaki H, Michikawa T, Furuichi T, Mikoshiba K (1999) Cooperative formation of the ligand binding site of the $\mathrm{IP}_{3}$ receptor by two separable domains. $\mathrm{J}$. Biol. Chem. 274: 328-334.

10. Norton WT (1974) Isolation of myelin from nerve tissue. Methods Enzymol. 31: 435-44.

11. Varki A (2007) Glycan-based interactions involving vertebrate sialic-acid-recognizing proteins. Nature 446: 1023-1029.

12. Rutishauser U, Landmesser L (1996) Polysialic acid in the vertebrate nervous system: a promoter of plasticity in cell-cell interactions. Trends in Neurosci. 19: 422-427.

13. Crocker PR (2002) Siglecs: sialic-acid-binding immunoglobulin-like lectins in cell-cell interactions and signaling. Curr. Opin. in Struc. Biol. 12: 609-615.

14. Filbin MT (2003) Myelin-associated inhibitors of axonal regenaration in the adult mammalian CNS. Nat. Rev. Neurosci. 4: 1-11.

15. Gee GV, Dugan AS, Tsomaia N, Mierke DF, Atwood WJ (2006) The role of sialic acid 
in human polyomavirus infections. Glycoconj. J. 23: 19-26. 


\section{Figure legends}

Fig. 1. Increased molecular size of Opalin in mouse brains from infancy to adulthood

(A) The precipitated ppt $2+3$ fractions $(2.5 \mu \mathrm{g} / \mathrm{lane})$ prepared from cerebellum and cerebrum of mice at P7, P14, P28, P56 and P180 were subjected to 12\% SDS-PAGE followed by western blot analyses using an anti-Opalin antibody. (B) The pure myelin fractions $(0.25$ $\mu \mathrm{g} / \mathrm{lane})$ of P23, P60 and P180 mouse brains were subjected to 15\% SDS-PAGE followed by western blot analyses using an anti-Opalin antibody. (C) A magnified image of the immunoreactive banding pattern. The pure myelin fractions $(0.3 \mu \mathrm{g} / \mathrm{lane})$ of P23, P60 and P180 mouse brains were separated by $15 \%$ SDS-PAGE. At P180, the amount of Opalin in the pure myelin fraction was decreased and the size of Opalin was increased, in comparison with those at P23 and P60. The upper dashed line (at $37 \mathrm{kDa}$ ) indicates around the top of the band from mouse brains at P180, and the lower dished line represents around the bottom of the band from young mouse brains at P23. Molecular weight standards in $\mathrm{kDa}$ are shown to the left of the blots.

\section{Fig. 2. Distribution of Opalin in mouse brains from infancy to adulthood}

(A)-(H) Parasagittal sections of mouse brains at P7 (A, E), P14 (B, F), P21 (C, G) and P180 $(\mathrm{D}, \mathrm{H})$ were immunostained for Opalin $(\mathrm{A}-\mathrm{D})$ and $\mathrm{MBP}(\mathrm{E}-\mathrm{H})$. It is notable that Opalin immunoreactivity peaks by $\mathrm{P} 21(\mathrm{C})$ and decreases in the cerebellum at P180 in comparison with the other regions such as the corpus callosum, anterior commissure, optic nerve and 
corticospinal tract (D). Such region-specific reduction of immunoreactivity in the cerebellum at P180 is not observed for $\operatorname{MBP}(\mathrm{H})$. Scale bar in panels $\mathrm{E}-\mathrm{H}, 1 \mathrm{~mm}$. (I)-(N) Coronal sections of mouse brains at P180 were immunostained for Opalin (I-K) and MBP $(\mathrm{L}-\mathrm{N})$. Opalin immunoreactivity decreases in the white mater regions of cerebellum at P180 in comparison with the other regions such as the corpus callosum, anterior commissure, optic nerve and corticospinal tract. Such region-specific reduction of immunoreactivity in the cerebellum at $\mathrm{P} 180$ is not observed for MBP. Scale bar in panels $\mathrm{L}-\mathrm{N}, 1 \mathrm{~mm}$. ac, anterior commissure; $c b w m$, cerebellar white matter; $c c$, corpus callosum; $c s t$, corticospinal tract (pyramidal tract); $f i$, fimbria of hippocampus; $f r$, fasciculus retroflexus; lo, lateral olfactory tract; $m s$, medullary stria of thalamus; oc, optic chiasm; ot, optic tract; $p f$, posterior funiculus; scwm, spinal cord white matter.

Fig. 3. Differential distribution of Opalin and MBP proteins and mRNAs in mouse spinal cords at juvenile (P15-P21) and adult (P180) stage

(A) Coronal sections of P15 (left panels) and P180 (right panels) mouse spinal cords were immunostained with anti-Opalin (top row) and anti-MBP (bottom row) antibody. At P180, Opalin immunoreactivity was relatively high levels in the $d c s t$, intermediate $\mathrm{n}$ the $f g$ and dms but low in the other white matter (WM) in the spinal cord. The patchy immunoreactivity for Opalin in the gray matter (GM) was almost comparable to that of MBP. Scale bar, $500 \mu \mathrm{m}$. (B) Higher magnification images of Opalin (top row)- or MBP 
(bottom row)-immunostained spinal cords from P21 mice. The GM panels of (B) show images around the anterior horn. Scale bars, WM-GM $100 \mu \mathrm{m}, \mathrm{WM}$ and GM $50 \mu \mathrm{m}$. (C) Coronal sections of P21 mouse spinal cords were analyzed by in situ hybridization with the riboprobes of Opalin (top row) and MBP (bottom row). Scale bar, $500 \mu \mathrm{m}$ and $100 \mu \mathrm{m}$. AH, anterior (ventral) horn; $c c$, central canal; $d c s t$, dorsal corticospinal tract; $d m s$, dosal median septum; $f c$, funiculus cuneatus; $f g$, funiculus gracilis; $P H$, posterior (dorsal) horn.

\section{Fig. 4. Sialylation levels and patterns of Opalin from weaning age to adulthood of mice}

(A) Increased sialylation of Opalin in adolescent mouse brain. Myelin fractions prepared from mouse brains at P23 (juvenile or weaning stage) and P180 (adult stage), were treated with a combination of three kinds of glycosidases: $O$-glycosidase, neuraminidase and $N$-glycosidase F, which remove $O$-glycans (but not sialylated $O$-glycans), sialic acids and $N$-glycans, respectively. Treated samples were analyzed by western blots using an anti-Opalin antibody. + and - indicate treatment and un-treatment, respectively. To obtain better immunoreactive signals, we separated larger amounts of P180 samples than P23 ones by SDS-PAGE: 1.5-fold in lanes 1-4, 2-fold in lanes 5-10, 1.5-fold in lanes 11-14 and 4.5-fold in lanes 15 and 16. Molecular weight standards in $\mathrm{kDa}$ are shown to the right. (B) Sialylation patterns of Opalin with aging. Myelin fractions from mouse brains at P23 and P180 were subjected to the affinity binding assay for three lectins Maackia amurensis lectin

II (MAL II = MAA2), Maackia amurensis agglutinin (MAA) and Sambucus nigra 
agglutinin (SNA), followed by western blot with anti-Opalin antibody. Input indicates samples without lectin treatment. $N$-Glyc, $N$-glycosidase; $O$-Glyc, $O$-glycosidase; NA, neuraminidase. 


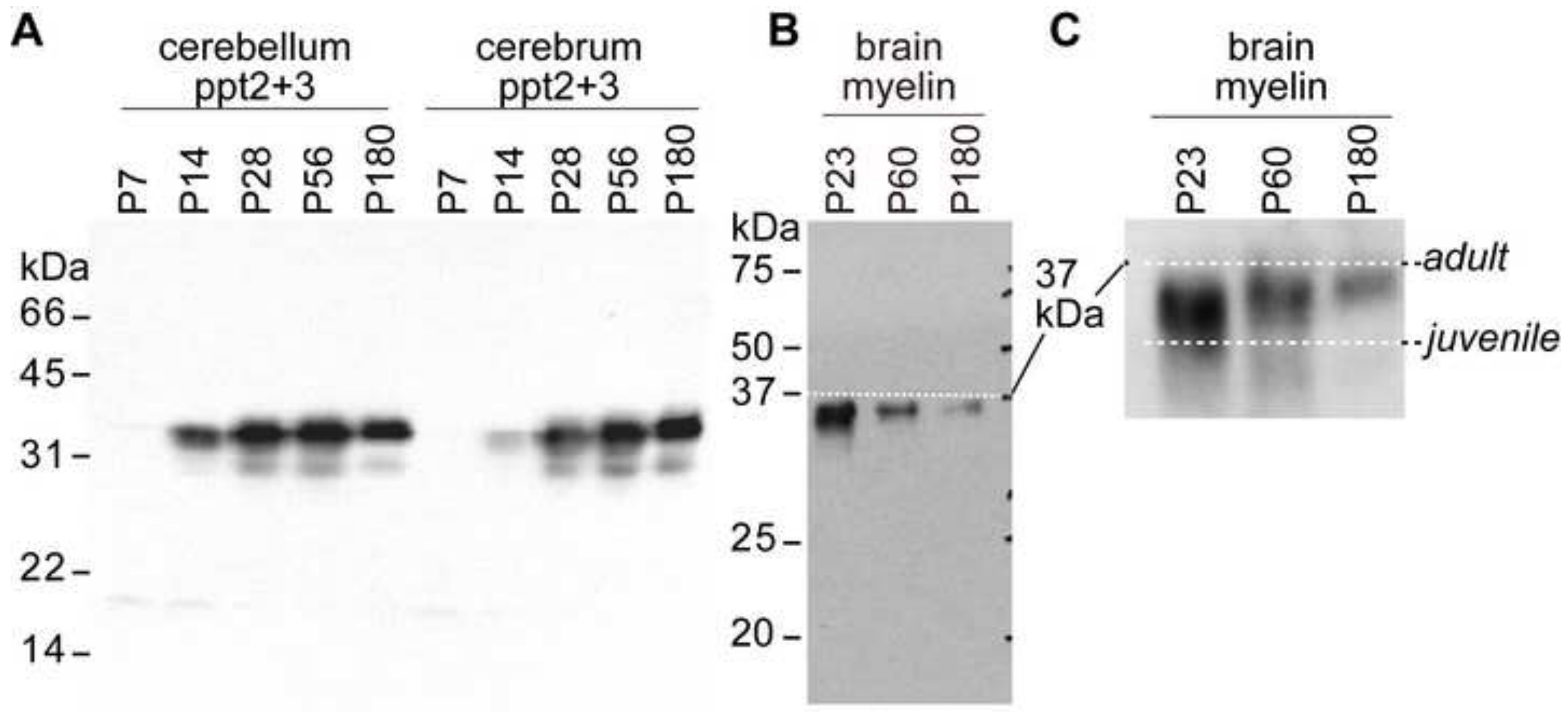


Opalin

A

P7

B
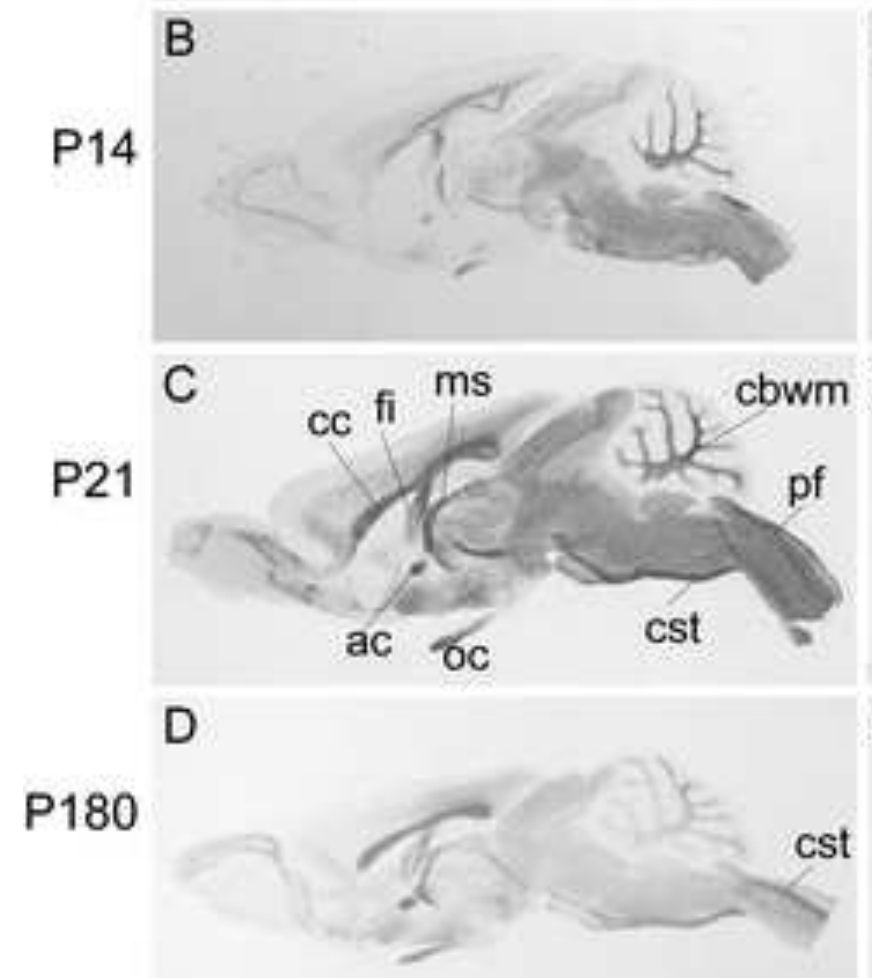

H

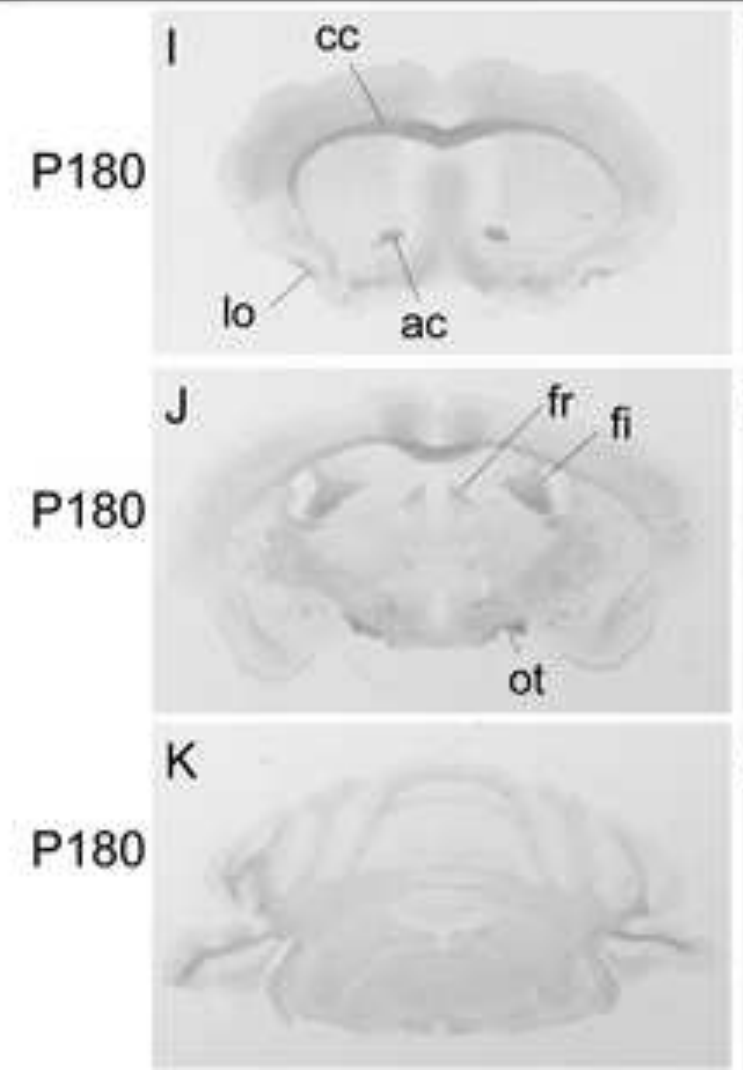

F
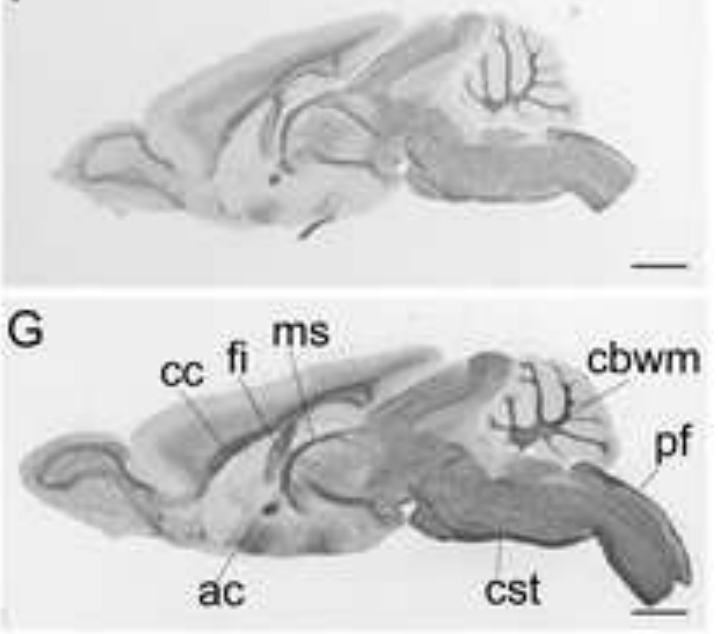

E

MBP
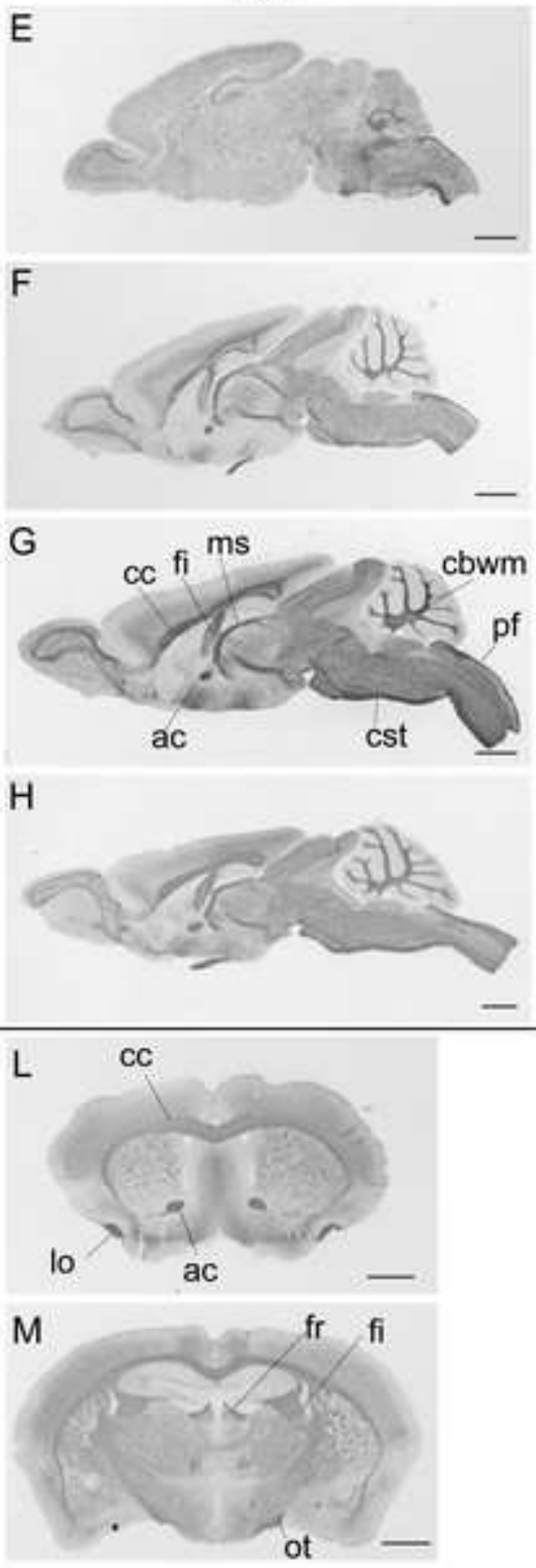

N cbwm

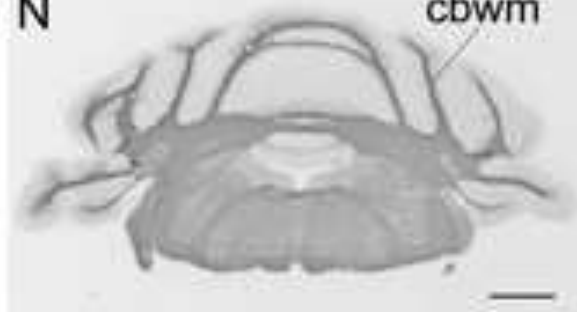


A

P15

P180

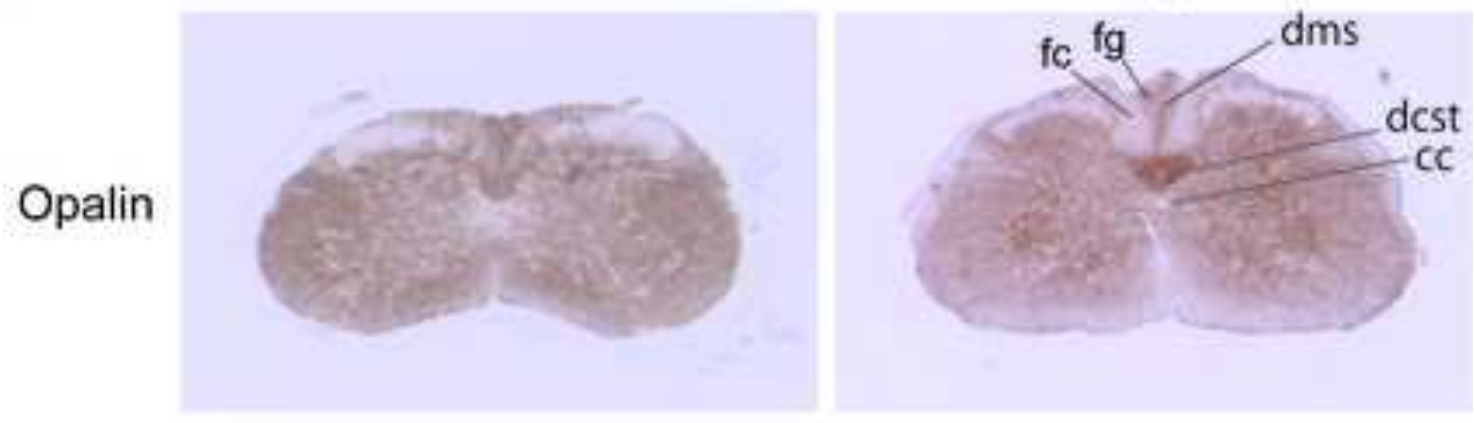

MBP

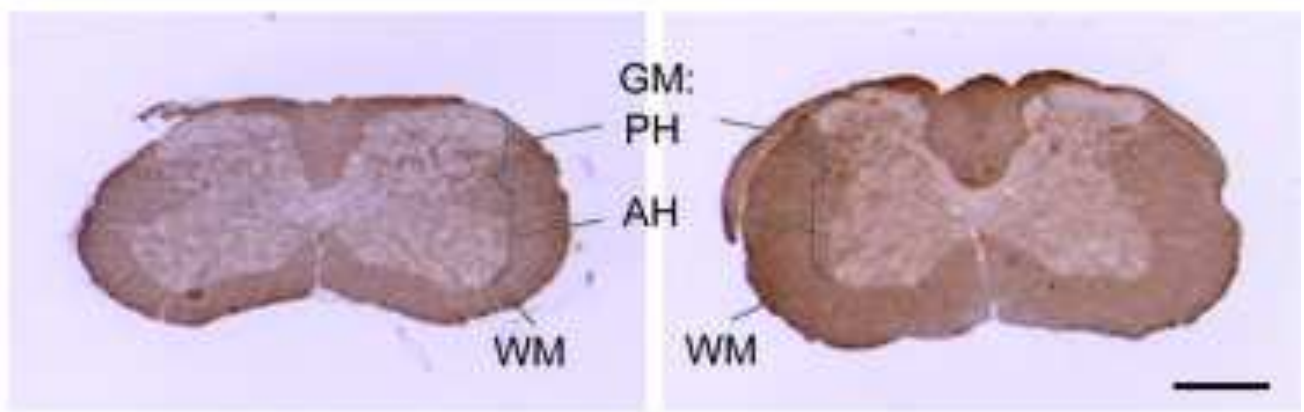

B

Opalin

(P21)
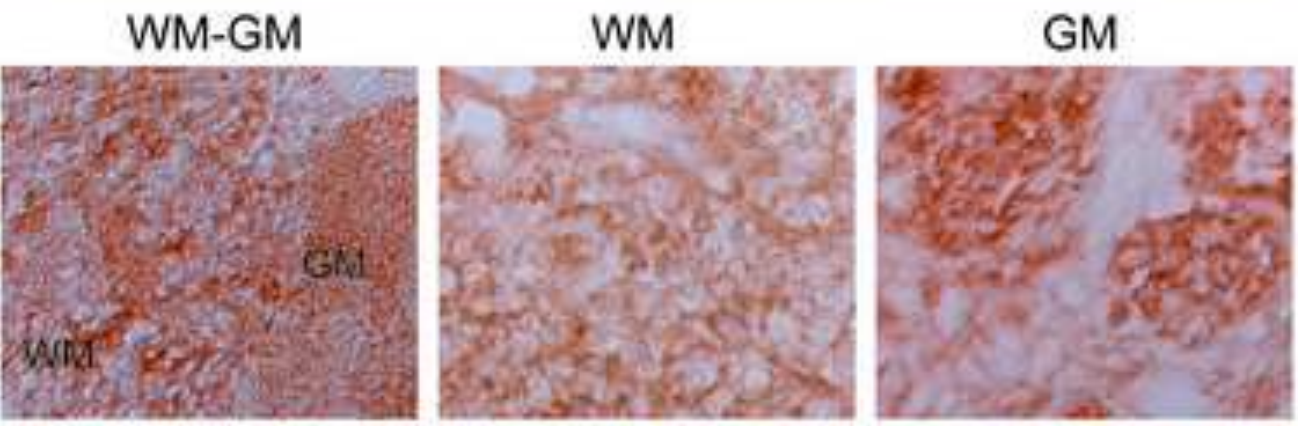

MBP

(P21)
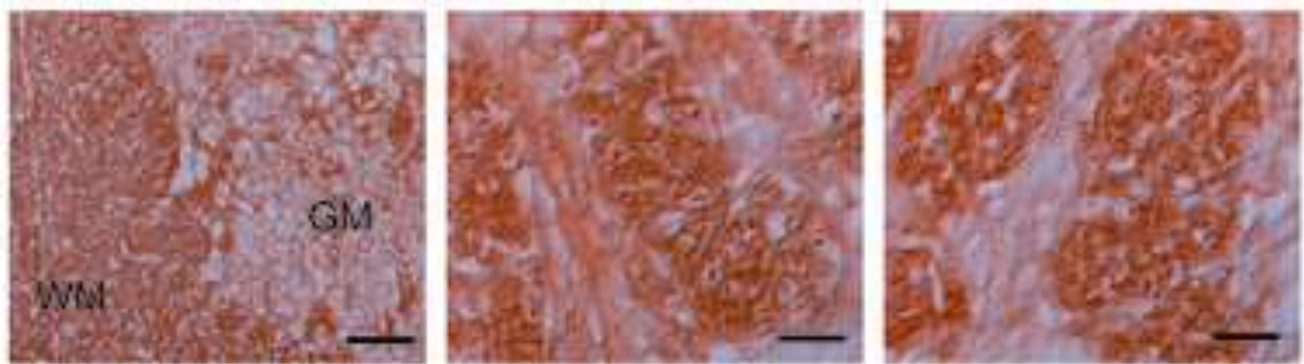

C

Spinal cord

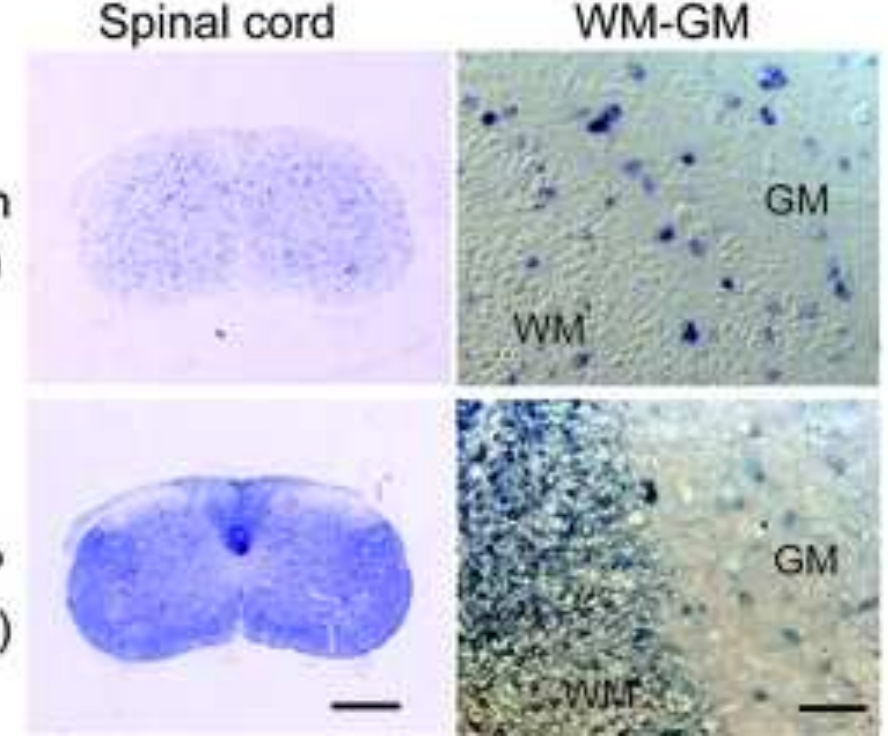

Opalin

(P21)

MBP

(P21) 
A

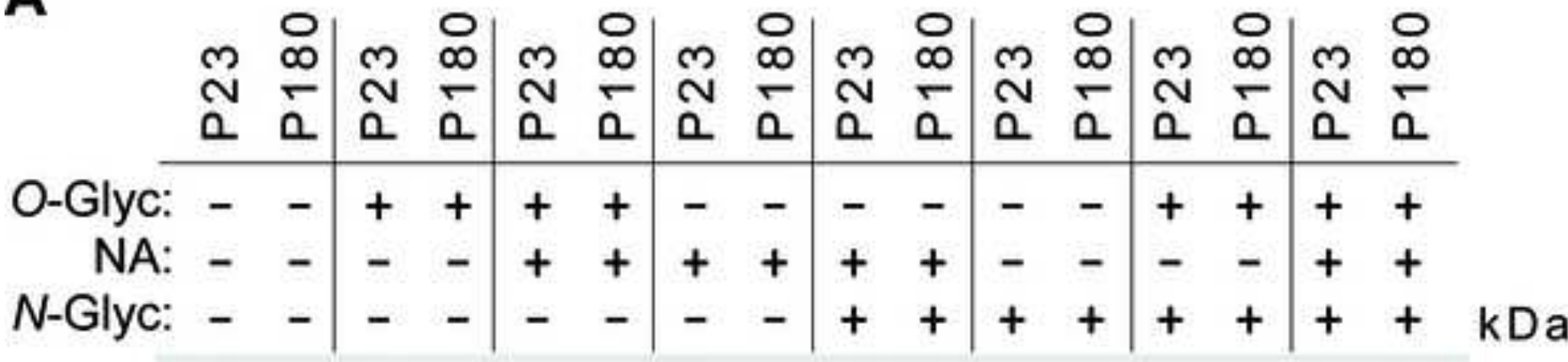

$$
\begin{aligned}
& \text { ton ens }
\end{aligned}
$$$$
\text { 6 * e }
$$

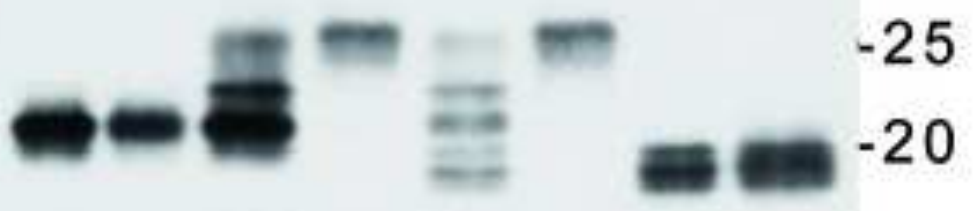$$
\begin{array}{llllllllllllllll}
1 & 2 & 3 & 4 & 5 & 6 & 7 & 8 & 9 & 10 & 11 & 12 & 13 & 14 & 15 & 16
\end{array}
$$

B

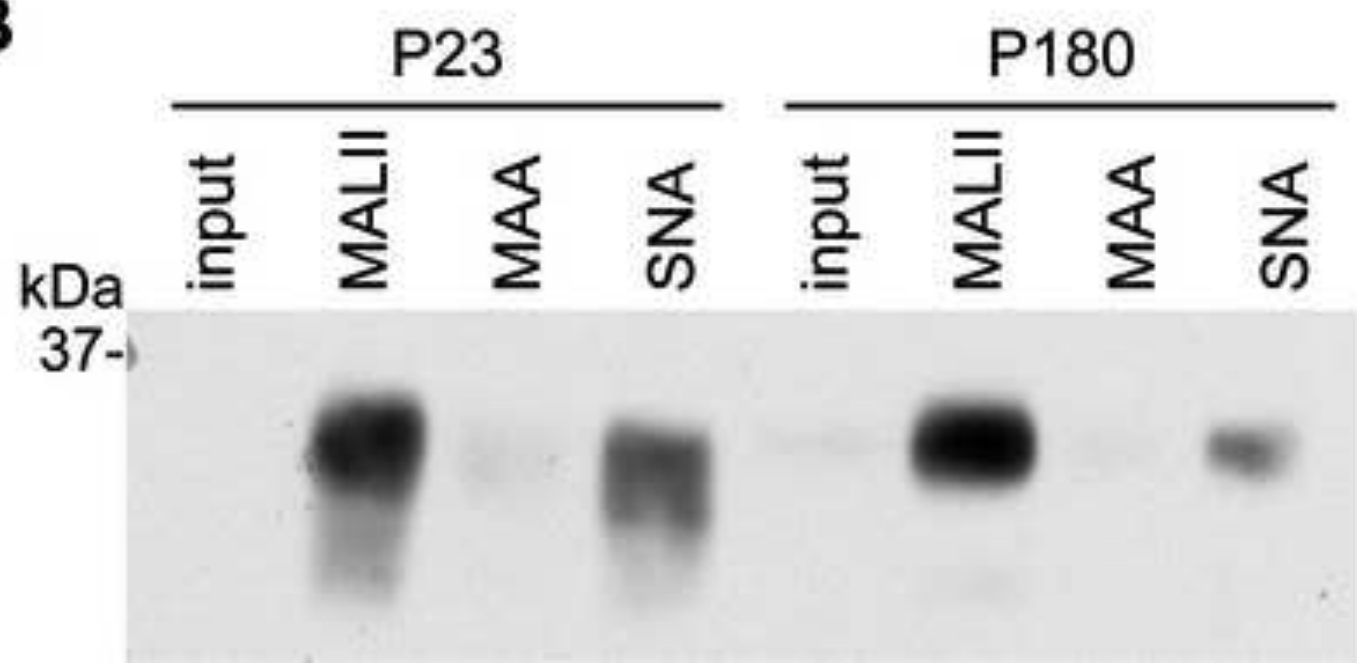

